\title{
Erratum
}

\section{Nucleation of Instability of the Meissner State of 3-Dimensional Superconductors}

\author{
Peter Bates ${ }^{1}$, Xing-Bin Pan ${ }^{2}$ \\ ${ }^{1}$ Department of Mathematics, Michigan State University, East Lansing, MI 48824, USA. \\ E-mail: bates@math.msu.edu \\ 2 Department of Mathematics, East China Normal University, Shanghai 200062, \\ People's Republic of China. E-mail: xbpan@math.ecnu.edu.cn
}

Received: 3 March 2008 / Accepted: 3 March 2008

Published online: 2 August 2008 - @ S Springer-Verlag 2008

Commun. Math. Phys. 276(3), 571-610 (2007)

In the above article, a condition on the domain is missing.

In the following lines, the word "simply-connected domain" should be replaced by "simply-connected domain without holes": Theorem 1, Proposition 2.1, Corollary 2.2, Lemma 2.3, Theorem 4.1, Theorem 5.1, Lemma 7.1, Lemma 7.3, Theorem 7.4, line 27 and line 30 on p. 577 (before (2.1)); footnote 8 on p. 577; line 4 on p. 578 (before (2.3)); line 15 on p. 605.

The reason for this is that on line 4 of p. 578, we quoted a result by Bolik-Wahl [BW]; see (2.3):

$$
\|\nabla \mathbf{B}\|_{C^{\alpha}(\bar{\Omega})} \leq C(\Omega, \alpha)\left\{\|\operatorname{div} \mathbf{B}\|_{C^{\alpha}(\bar{\Omega})}+\|\operatorname{curl} \mathbf{B}\|_{C^{\alpha}(\bar{\Omega})}+\|v \times \mathbf{B}\|_{C^{1+\alpha}(\partial \Omega)}\right\},
$$

which requires the domain to have no holes; see [BW], Theorem 2.1. In the proof of Proposition 2.1 in our paper, when $k \geq 1$, we used the inequality (2.3). Since, in the proof of our main theorems, we used Proposition 2.1, the domain should have no holes. In the proof of (2.2) we also require the domain to have no holes.

\section{Reference}

[BW] Bolik, J., von Wahl, W.: Estimating $\nabla \mathbf{u}$ in terms of $\operatorname{div} \mathbf{u}$, curl $\mathbf{u}$, either $(v, \mathbf{u})$ or $v \times \mathbf{u}$ and the topology. Math. Methods Appl. Sci. 20, 737-744 (1997)

Communicated by P. Constantin

The online version of the original article can be found under doi:10.1007/s00220-007-0335-y. 